\title{
Anaemia Among Refugees Pregnant Women in Gaza Strip: Perceptions, Awareness and Opinions and Attitudes
}

\author{
Marwan Mosleh ${ }^{1, *}$, Koustuv Dalal ${ }^{2}$ \\ ${ }^{1}$ Ministry of Health, Gaza Strip, Palestine \\ ${ }^{2}$ School of Health and Education, University of Skovde, Skovde, Sweden
}

Email address:

marwanmoon@hotmail.com (M. Mosleh)

${ }^{*}$ Corresponding author

To cite this article:

Marwan Mosleh, Koustuv Dalal. Anaemia Among Refugees Pregnant Women in Gaza Strip: Perceptions, Awareness and Opinions and Attitudes. Journal of Gynecology and Obstetrics. Vol. 7, No. 4, 2019, pp. 109-115. doi: 10.11648/j.jgo.20190704.13

Received: May 23, 2019; Accepted: June 25, 2019; Published: July 10, 2019

\begin{abstract}
Anaemia in pregnancy is the most common public health concern in both developed and developing worlds like Palestine. Little is known about this significant health issue. Therefore, the purpose of this study was to investigate the perception, opinions and attitudes of anaemia among refugees pregnant women in Gaza strip. A cross-sectional study was employed among a random sample of 164 women or subjects, with a response rate of $81 \%$. The survey was conducted at UNRWA antenatal health centers at refugee camps in Gaza strip. The study was approved by United Nations Relief and Work Agency (UNRWA) health department. The study elucidated that a higher rate of women were knowledgeable and had ideas on anaemia, however, some women had inadequate knowledge on anaemia during pregnancy. There were a concern and misconception regarding some issues on anaemia such as; lack of knowledge on the effect of drinking tea on iron absorption; and on the side effects of iron pills during pregnancy. Highly positive opinions and attitudes toward anaemia were observed in most women. The study found that a high proportion of women were dissatisfied with the volume of health education sessions on anaemia at UNRWA antenatal health centers and many women had not attended or joined such sessions. In general, the study declined to find a significant differences regarding anaemia between women with and those without health education sessions on anaemia. Finally, the study suggests that, an effective and a clear health education strategy is needed in order to improve women perceptions, awareness, opinions and attitudes regarding anaemia in Palestine. Further researches are important and needed in this scope.
\end{abstract}

Keywords: Perceptions, Anaemia, Refugees, Pregnant Women, UNRWA, Gaza Strip

\section{Introduction}

Anaemia is known as a major public health concern globally, in both developed and developing countries with a major impacts for individuals health, economic and social development. It is most prevalent in pregnancy [1]. Aanemia is a common nutritional problems worldwide mainly in developing world [2]. It is identified a leading cause of maternal morbidity, mortality and problematic birth outcomes in developing worlds. Anaemia and iron deficiency anaemia affect about 3.5 billion people globally, with 110,000 maternal deaths attributable to iron deficiency annually [3]. It is defined by World Health Organization (WHO), as " a condition in which the number of red blood cells (RBCs) or their oxygen-carrying capacity is insufficient to meet physiologic needs, which varies by age, ex, altitude, smoking, and pregnancy status" [4]. It is caused due to several factors; the most common cause is iron deficiency which is responsible for about half of cases [5]. Although, anemia was addressed as global public health concern recently, however, no dramatic progress was observed and its prevalence still high nationally and internationally [6]. Indeed, women demand is increased for iron from puberty to menopause due to excess blood loss during menstruation stage. Thus, iron deficiency is most common among women of reproductive age in the world [7, 8]. Anaemia is therefore considered one of the most common problem complicating maternal women worldwide especially in low-income countries [9]. Anaemia in pregnancy is defined by WHO as 
"hemoglobin concentration less than $11 \mathrm{~g} / \mathrm{dl}$ " [10]. During pregnancy the requirements of iron is increased among women due to physiological change and high demands of iron for the development and growing of the placenta and fetus [11]. Since, it was estimated that anaemia prevalence among pregnancy were $18 \%$ in developed worlds, whereas it is between $35-75 \%$ in developing worlds [12].

In Palestine, anaemia remains a growing public health problem among women of reproductive age in spite of all efforts, prevention programs and intervention conducted by UNRWA and Ministry of Health $(\mathrm{MOH})$ to tackle the disease in Gaza strip [13, 14]. Where, several studies emphasized that Gaza strip still has a higher rate of anaemia in pregnancy comparing with neighboring regions [15]. Since, the figures showed that anaemia prevalence among pregnancy was $45.10 \%$ in Gaza strip while $20.60 \%$ in the West bank were anaemic in 2009 [16]. The prevalence of anaemia in Gaza strip had the highest rate in pregnancy and is doubled those reported in Europe [15]. Poor misconception and lack of awareness and knowledge on anaemia could be a part of explanation to this significant health problem in pregnancy. The study tried to bridge the gap in knowledge and literature about anaemia in Palestinian territory. Therefore, the purpose of this study was to describe and analyze perceptions, opinions and attitudes among refugees pregnant women in Gaza strip.

\section{Methods and Subjects}

\subsection{Study Setting and Subjects}

The study was undertaken at four UNRWA antenatal health centers in the refugees camps of Gaza strip. As UNRWA is the main provider of health services and humanitarian aids for the refugees in Gaza strip and pregnancy in particular [17, 18]. All registered pregnant women who attending UNRWA antenatal health centers were the target population for this study. A total of 164 were randomly selected and included in the survey.

\subsection{Study Design}

This was a cross-sectional study was employed among refugees pregnant women who attended UNRWA antenatal healthcare center in Gaza strip.

\subsection{Tool of the Study}

Administered questionnaire was specially developed and piloted for this study, the questionnaire was then revised and adjusted in a way to ensure the reliability and clarity of the questions. The interviewer has described to women the purpose and importance of the study. The women were also informed that their answers will be secret and confident and their right to withdraw or refuse to participate in the survey. The sheet included four sections; the $1^{\text {st }}$ section included socio-demographic information about (age, education, occupation, number of children, income, attending health sessions); the $2^{\text {nd }}$ section was about knowledge and awareness on anaemia (concepts, nutrition, iron supplement, side effects, signs and symptom and effect of tea on iron absorption); the $3^{\text {rd }}$ section was about opinions and attitudes regarding anaemia; and the section $4^{\text {th }}$ was about health education on anaemia.

\subsection{Statistical Analysis}

Data were analyzed using SPSS version 22. Frequency and proportions were analyzed. Cross-tabulation was computed, with chi-square $\left(\mathrm{X}^{2}\right)$ test to compare categorical variables. Differences and associations were considered to be significant, when ( $\mathrm{p}$-value $<0.05)$.

\subsection{Ethical Considerations}

Ethical approval was obtained from ethical committee at UNRWA health department. Oral consent was also considered from all surveyed women at UNRWA antenatal health centers.

\section{Results}

The study showed that women ages ranged from less than 20 to more than 30 years, a higher proportion of women was of age group of 20-25 year, the maximum percentage of them had a secondary school certificate and more than a third had a diploma or university degree. Plenty of women were housewives and almost half of them had seven children or more. The maximum number of women were from low and middle-income background, whereas, the lowest percentage was from high-income level and most of them had attended health education sessions (table 1).

Table 1. Sociodemographic profile of study samples $(n=164)$.

\begin{tabular}{ll}
\hline Items & Frequency (\%) \\
\hline Age & $14(8.5 \%)$ \\
Less than 20 & $57(34.8 \%)$ \\
$20-25$ & $41(25.0 \%)$ \\
$26-30$ & $52(31.7 \%)$ \\
More than 30 & \\
Literacy level & $34(20.7 \%)$ \\
Primary & $69(42.1 \%)$ \\
Secondary & $17(10.4 \%)$ \\
Middle education/diploma & $44(26.8 \%)$ \\
Under and post graduate & \\
Occupation & $135(82.3 \%)$ \\
No & $29(17.7 \%)$ \\
Yes & \\
Children number & $26(15.9 \%)$ \\
No children & $23(14.0 \%)$ \\
$<3$ & $41(25.0 \%)$ \\
$3-6$ & $41(25.0 \%)$ \\
$7-10$ & $33(20.1 \%)$ \\
$>10$ & \\
Economic status & $67(40.9 \%)$ \\
Low & $52(31.7 \%)$ \\
Middle & $45(27.4 \%)$ \\
High & \\
Yttending health sessions & $112(68.3 \%)$ \\
No & $52(31.7 \%)$ \\
\hline
\end{tabular}


The majority of women $68 \%$ had attended health education sessions, while few women $32 \%$ had not attended such sessions on anaemia. The highest percentage of women who attended health education sessions were from age group 2630 years or above. The results demonstrated that attending health education sessions seems to be increased by age. The findings also showed that attendance rate was increased gradually by increasing education level. In addition, nonattendance health education sessions has also been observed higher among those who were less-educated than upper educated women. A higher proportion of labourers than housewives had attended health education sessions. Women who had first-onset of pregnancy and those who had few children were more likely to attend sessions than women who had many births. Women from middle and high-income background were more likely to attend sessions than women from low-income background. However, one should note that there were no significant relationship in any case (table 2).

Table 2. Rate of women who had access to health education sessions by sociodemographic.

\begin{tabular}{|c|c|c|c|c|}
\hline \multirow{2}{*}{ Items } & \multicolumn{2}{|c|}{ Attending health education sessions } & \multirow{2}{*}{ Total } & \multirow{2}{*}{ p-value } \\
\hline & Yes $n=112(68 \%)$ & No $\mathrm{N}=52(32 \%)$ & & \\
\hline \multicolumn{5}{|l|}{ Age } \\
\hline Less than 20 & $9(64 \%)$ & $5(36 \%)$ & 14 & \multirow{4}{*}{0.695} \\
\hline $20-25$ & $36(63 \%)$ & $21(37 \%)$ & 57 & \\
\hline $26-30$ & $30(73 \%)$ & $11(27 \%)$ & 41 & \\
\hline More than 30 & $37(71 \%)$ & $15(29 \%)$ & 52 & \\
\hline \multicolumn{5}{|l|}{ Literacy level } \\
\hline Primary & $18(53 \%)$ & $16(47 \%)$ & 34 & \multirow{4}{*}{0.143} \\
\hline Secondary & $48(70 \%)$ & $21(30 \%)$ & 69 & \\
\hline Middle education/diploma & $12(71 \%)$ & $5(29 \%)$ & 17 & \\
\hline Under and post graduate & $34(77 \%)$ & $10(23 \%)$ & 44 & \\
\hline \multicolumn{5}{|l|}{ Occupation } \\
\hline No & $90(67 \%)$ & $45(33 \%)$ & 135 & \multirow{2}{*}{0.334} \\
\hline Yes & $22(76 \%)$ & $7(24 \%)$ & 29 & \\
\hline \multicolumn{5}{|l|}{ Children number } \\
\hline No children & $19(73 \%)$ & $7(27 \%)$ & 26 & \multirow{5}{*}{0.216} \\
\hline$<3$ & $16(70 \%)$ & $7(30 \%)$ & 23 & \\
\hline $3-6$ & $32(78 \%)$ & $9(22 \%)$ & 41 & \\
\hline $7-10$ & $27(66 \%)$ & $14(34 \%)$ & 41 & \\
\hline$>10$ & $18(55 \%)$ & $15(45 \%)$ & 33 & \\
\hline \multicolumn{5}{|l|}{ Economic status } \\
\hline Low & $43(64 \%)$ & $24(36 \%)$ & 67 & \multirow{3}{*}{0.583} \\
\hline Middle & $38(73 \%)$ & $14(27 \%)$ & 52 & \\
\hline High & $31(69 \%)$ & $14(31 \%)$ & 45 & \\
\hline
\end{tabular}

$(\mathrm{p}<0.05)=$ significant value.

The findings pointed that the highest proportion of women $>90 \%$ were aware that diet rich in iron is necessary for pregnancy and a high percentage of them were aware that anaemia is caused due to malnutrition with inadequate iron. Furthermore, a highly percentage of women were knowledgeable and aware that severe malnutrition is a major risk factors for anaemia and most women were aware that iron supplementation is necessary during pregnancy. Highly proportion of women knew that "anaemia is meant as iron deficiency in the blood" and they had general knowledge on anaemia "perception and its diagnosis by blood test".
Moreover, a high proportion of women were aware that "anaemia has adverse impact on women and fetus health". On the other hand, the findings showed that there was gradual drop of women knowledge and awareness toward other questions particularly on "the effect of tea with meals on iron absorption", where the data have shown that many women were not aware and less knowledgeable regarding "tea with meals decrease iron absorption in the blood". In addition, the results have shown a lack of knowledge and misconception regarding "the side effects of iron pills", where knowledge as also low (table 3).

Table 3. Proportion of samples as classified by perception and awareness, opinions and attitudes, and health education sessions on anaemia.

\begin{tabular}{|c|c|c|c|}
\hline Items & Completely agree & Partially agree & Disagree \\
\hline \multicolumn{4}{|l|}{ Perception and awareness } \\
\hline Diet rich in iron is necessary for pregnancy & $93.3 \%$ & $6.1 \%$ & $0.6 \%$ \\
\hline Anaemia is caused due to malnutrition with insufficient iron & $89.6 \%$ & $9.1 \%$ & $1.2 \%$ \\
\hline Severe malnutrition is a major risk factor of anaemia in prgnancy & $87.2 \%$ & $12.2 \%$ & $0.6 \%$ \\
\hline Iron pills supplementary is necessary during pregnancy & $86.0 \%$ & $9.8 \%$ & $4.3 \%$ \\
\hline Anaemia is meant as iron deficiency in the blood & $82.9 \%$ & $11.6 \%$ & $5.5 \%$ \\
\hline Anaemia is diagnosed by testing of $\mathrm{Hbg}$ in the blood & $82.3 \%$ & $15.2 \%$ & $2.4 \%$ \\
\hline Anaemia has adverse effect on pregnant $\&$ festus health & $82.3 \%$ & $14.0 \%$ & $3.7 \%$ \\
\hline Dizziness, tiredness, and weakness are significant signs of anaemia during pregnancy & $73.2 \%$ & $25.0 \%$ & $1.8 \%$ \\
\hline Tea with meals increase iron absorption in the blood & $24.4 \%$ & $14.0 \%$ & $61.6 \%$ \\
\hline Iron pills have side effects may occur during pregnancy & $39.0 \%$ & $34.1 \%$ & $26.8 \%$ \\
\hline
\end{tabular}




\begin{tabular}{|c|c|c|c|}
\hline Items & Completely agree & Partially agree & Disagree \\
\hline \multicolumn{4}{|l|}{ Opinions and attitudes } \\
\hline Adherence of having iron pills is necessary during pregnancy & $91.5 \%$ & $6.7 \%$ & $1.8 \%$ \\
\hline Adherence to have rich diet with iron is necessary in pregnancy & $90.9 \%$ & $7.3 \%$ & $1.8 \%$ \\
\hline Anaemia threaten pregnant and fetus health & $87.2 \%$ & $9.1 \%$ & $3.7 \%$ \\
\hline Regular antenatal visits are necessary for pregnancy & $84.8 \%$ & $11.6 \%$ & $3.7 \%$ \\
\hline Adherence to have iron pills should be after Dr. order & $70.1 \%$ & $19.5 \%$ & $10.4 \%$ \\
\hline Having iron pills without DR. order cause side effects & $51.2 \%$ & $32.3 \%$ & $16.5 \%$ \\
\hline \multicolumn{4}{|l|}{ Health education sessions on anaemia } \\
\hline Health education sessions are important for pregnancy & $88.4 \%$ & $10.4 \%$ & $1.2 \%$ \\
\hline Pregnant women is on a need to health education sessions & $84.1 \%$ & $14.6 \%$ & $1.2 \%$ \\
\hline Regular health education on anaemia can reduce the prevalence of anaemia among pregnancy & $82.6 \%$ & $14.6 \%$ & $3.0 \%$ \\
\hline Adherence to attend health education sessions are necessary during pregnancy & $81.1 \%$ & $17.1 \%$ & $1.9 \%$ \\
\hline UNRWA has adequate health education sessions on anaemia particularly during pregnancy & $31.1 \%$ & $58.5 \%$ & $10.5 \%$ \\
\hline
\end{tabular}

In regard to women opinions and attitudes, the findings revealed that the highest proportion of women were aware that "adherence of having iron supplementation is necessary during pregnancy", and a high percentage of women of them were aware that "adherence to have sufficient diet rich of iron is necessary". Since, the findings showed that women attitudes towards "iron supplementation, diet rich of iron, anaemia risk on women and fetus, and regular antenatal visits to clinics" seems to be positively high an most women completely agreed and encouraged it. Hence, a gradual change in women opinions and attitudes was observed in some questions and reached the lowest specifically regarding "the problem of having iron pills without prescription", where, only about half of women were knowledgeable that "taking iron pills without physicians prescription may cause undesirable side effects" (table 3). The study showed that the highest proportion of women were aware and completely agree that health education sessions on anaemia are important for antenatal care, followed by the questions about the need of health education sessions. Most women were aware of "the importance of regular health education sessions to reduce the prevalence of anaemia among pregnant women" and "the necessity of attending such sessions especially during pregnancy". In contrast, the study found that high proportion of women were dissatisfied with the number of sessions, where less than one third were completely satisfied with the number of sessions provided by UNRWA antenatal centers (table 3 ).

Finally, the study indicated that there was no significant differences of awareness between women who attended health education sessions and those who did not attend such sessions, except only one variable was statistically significant $(p<0.05)$, where the figures indicated that women with health education sessions were more aware than women without health education sessions regarding "the need of taking iron pills" (table 4).

Table 4. Comparison of perception and awareness between women with and without health education sessions on anaemia.

\begin{tabular}{|c|c|c|c|c|c|c|c|}
\hline \multirow{3}{*}{ Items } & \multicolumn{6}{|c|}{ Health education sessions } & \multirow{3}{*}{ p-value } \\
\hline & \multicolumn{2}{|c|}{ Yes $n=112(68 \%)$} & \multicolumn{4}{|c|}{ No n=52 (32\%) } & \\
\hline & $\begin{array}{l}\text { comp. } \\
\text { agree }\end{array}$ & $\begin{array}{l}\text { partially } \\
\text { agree }\end{array}$ & disagree & $\begin{array}{l}\text { comp. } \\
\text { agree }\end{array}$ & $\begin{array}{l}\text { partially } \\
\text { agree }\end{array}$ & disagree & \\
\hline \multicolumn{8}{|l|}{ Perception and awareness of anaemia } \\
\hline Severe malnutrition is a major risk factor of anaemia & $87.5 \%$ & $12.5 \%$ & - & $86.5 \%$ & $11.5 \%$ & $2.5 \%$ & 0.335 \\
\hline Anaemia is caused due to malnutrition with inadequate iron & $91.0 \%$ & $8.0 \%$ & $0.9 \%$ & $86.5 \%$ & $11.5 \%$ & $2.0 \%$ & 0.648 \\
\hline Iron pills supplementary is necessary in pregnancy & $88.5 \%$ & $8.0 \%$ & $4.0 \%$ & $81.0 \%$ & $13.0 \%$ & $6.0 \%$ & 0.425 \\
\hline Diet rich with iron is necessary for pregnancy & $95.0 \%$ & $5.0 \%$ & - & $90.0 \%$ & $8.0 \%$ & $2.0 \%$ & 0.281 \\
\hline $\begin{array}{l}\text { Tea with meals increase iron absorption in the blood } \\
\text { Opinions \& attitudes regarding anaemia }\end{array}$ & $26.0 \%$ & $12.5 \%$ & $62.0 \%$ & $21.0 \%$ & $17.0 \%$ & $62.0 \%$ & 0.634 \\
\hline Having iron pills without dr. order may cause side effects & $53.5 \%$ & $34.0 \%$ & $12.5 \%$ & $46.0 \%$ & $29.0 \%$ & $25.0 \%$ & 0.133 \\
\hline Anaemia threaten pregnancy and fetus health & $89.0 \%$ & $8.0 \%$ & $3.0 \%$ & $83.0 \%$ & $11.0 \%$ & $6.0 \%$ & 0.454 \\
\hline $\begin{array}{l}\text { Adherence of having iron pills supplement is necessary in } \\
\text { pregnancy }\end{array}$ & $93.0 \%$ & $7.0 \%$ & - & $88.0 \%$ & $6.0 \%$ & $6.0 \%$ & $0.036 *$ \\
\hline $\begin{array}{l}\text { Regular antenatal visits are necessary for pregnancy } \\
\text { Importance of health education sessions }\end{array}$ & $85.0 \%$ & $11.6 \%$ & $3.6 \%$ & $84.5 \%$ & $11.5 \%$ & $4.0 \%$ & 0.996 \\
\hline UNRWA has adequate health education sessions on anaemia & $37.0 \%$ & $54.0 \%$ & $9.0 \%$ & $19.0 \%$ & $67.0 \%$ & $13.0 \%$ & 0.076 \\
\hline $\begin{array}{l}\text { Adherence to attend health education sessions are necessary } \\
\text { during pregnancy }\end{array}$ & $83.0 \%$ & $16.0 \%$ & $0.9 \%$ & $77.0 \%$ & $19.0 \%$ & $4.0 \%$ & 0.356 \\
\hline
\end{tabular}

${ }^{*}(\mathrm{p}<0.05)=$ significant value.

\section{Discussions}

Anaemia in pregnancy is a public health concern in
Palestine especially in Gaza strip. It can be classified as moderate in the West bank and severe in Gaza strip [15]. Recent studies summarized that anaemia rate is so high among pregnant women in Gaza strip comparing with the 
West bank and neighboring regions with a sharp increase in the latest years [16]. This increase might be referred to several factors in this problematic context such as; low socioeconomic background, insufficient medical instruments and health services, malnutrition, high level of unemployment, or might be due to lack of awareness and poor knowledge on anaemia among pregnancy.

Lack of awareness and knowledge on anaemia could be a part for the explanation to this significant health problem among pregnant women who attending UNRWA clinics for healthcare. It is worth mentioned that studies have not been published recently on perception and awareness of refugees pregnant women on anaemia in Gaza strip. A lack of such studies should receive particular attention from authorities in Gaza strip including (UNRWA, $\mathrm{MOH}$, and private sectors) to address the problems and exploring the underlying reasons behind that. Therefore, this study was conducted to describe and analyze the conception, knowledge, opinions and attitudes of anaemia among pregnant women at UNRWA antenatal health centers in Gaza strip. In this study, a high proportion of women was of age 20-25 years and the lowest was of age group less than 20 , indicating that women in this age are still in their best years of reproductive age and women of more than thirty indicates that women in this age also still capable of childbearing. In Palestinian females, the popular age for childbirth are ranged between 20-25 years. The study reported absence of women who did not attend primary school and a rather high educational level. Since, it is common in Palestinian females to pursue a secondary school and many girls continue to upper education. The evidences have shown that the literacy rate is high among girls in Palestine and has increased from $77 \%$ in 1995 to $89.8 \%$ in 2006 [19]. Despite the fact that country was identified as lower-middle-income since the mid-1990s, it performs at an upper middle-income country level in terms of education [20]. In regard of children number, it is obvious that most women were more likely to have many children. These findings were in line with findings presented in the annual reports of the United Nations, which referred it to prolonged humanitarian crises in Palestine, particularly in the Gaza strip and West bank and the desire of families to have more children or might be pressured to be more pregnant in order to compensate the slashed population and replacing individuals who were missed in the conflict events in the latest years in the aim to keep population balance and maintaining social and cultural identity [21]. It was revealed that educated women seemed more likely to attend health education sessions at UNRWA health centers than lesseducated women. The outcome of this study indicated that the attendance rate of health education sessions is increased gradually with advancing level of education comparing with those of lower education level. These conclusions were in line with evidences in previous studies of Gaza strip. Where, the evidences showed that that there was a direct relationship between educational level of women and attending antenatal health centers [9] However, the study failed to find a significant differences among different education level regarding attendance of health education sessions. The strongest reasons behind that, might be due to a smaller sample size of this study. It was also observed that women who had many children seemed unlikely to attend health education sessions comparing with women who had few or no children. From my point of view, I think this could be returned to the fact that women who had many births knew a lot about anaemia from their previous pregnancies compared to those who became pregnant for the first time or had new pregnancy. Therefore, new pregnancies women seemed more eager to attend health education sessions compared with women who had many children. This might be associated with the fact that medical staff or midwives at health centers, gave more attention to women who had new pregnancies rather than women who had many birth. Majority of women were housewives and the highest percentages of them were from low-income level and most of them attended health education sessions. The fact is that most of Palestinian refugees live under poverty line and survive on UNRWA relief and assistance [22]. The evidences have been observed from the survey performed in the latest years, which showed that more than half of Gaza households were living in poverty $56 \%$, while in the West bank households poverty rate was $48 \%$, and the study also showed that Palestinian refugees remain more vulnerable to poverty than non-refugees [23]. In respect to knowledge and awareness, our study clearly demonstrated that the refugees women were aware and knowledgeable of anaemia, however, some women still lack of knowledge on some specific issues of anaemia. For example, they seemed more likely to have good knowledge about the importance of diet rich of iron during pregnancy and were also knowledgeable that malnutrition causes anaemia and knew the importance of iron supplementary during pregnancy periods. The findings are consistent with the findings in other study performed in among pregnant women in Nablus in the West bank of Palestine which revealed that pregnant women had a high knowledge about anaemia in general especially regarding anaemia concept, anaemia symptoms, causes of anaemia, diet rich in iron and iron supplementation [24]. Highly level of awareness regarding anaemia in pregnancy might be associated with upper educational level. Despite UNRWA efforts and interventions to enhance women perception and awareness, pregnant women still unaware and lack of information of some aspects of great importance. Since, some women were less knowledgeable or had misunderstanding regarding the side effects of iron pills supplementary during pregnancy. This might be referred to one or several reasons such as; UNRWA sessions may lack of information on specific issues of anemia e.g. the potential side effects of iron pills that may occur during pregnancy (nausea, vomiting, abdominal discomfort and constipation) [25]. Other possible explanation is that health sessions might be irregular, insufficient, not well-organized and sometimes lack trained staff. Misconception is also obvious regarding the effect of drinking tea with meals on iron absorption. Where, the study provided evidence that $24 \%$ of women believed that drinking 
tea with meals increase iron absorption. In Palestinian territories, tea is a familiar drinking habit among people as well as pregnancies, however, some women is lacking information regarding the negative effect of drinking tea with meals. A poor practice and attitudes among women did not reflect the knowledge level regarding drinking tea and its inhibition role of iron absorption. These negative practices and attitudes may need more attention by UNRWA and other specialized stakeholders in Gaza strip. Poor knowledge might be linked with some factors such as; health education sessions are not enough to emphasizing this problem. Other women perhaps believe that health education sessions at UNRWA clinics are useless and just wasting their times. In regard to opinions and attitudes, the evidences showed that a highly positive opinions and attitudes were found toward; iron pills supplement during pregnancy and also regarding the necessity of sufficient diet rich of iron. Furthermore, highly positive and attitudes were observed on the questions which revealed that anaemia threats pregnant and fetus health and also regarding the importance of regular antenatal visits of pregnant women. These findings are in recur with the results of the West bank study [24]. The highly positive and attitudes among these women may also be reflected from their high level of knowledge and awareness of anaemia and their educational background. Nevertheless, the study demonstrated that only half of women completely agreed that taking iron pills continuously and regularly without prescription may cause undesired side effects. Women may be believe that overuse of iron pills will be very useful to their health regardless specialist prescription or medical order. I think this issue needs particular consideration and attention from UNRWA. The survey provided evidences that a high proportion of women were dissatisfied with the volume of UNRWA health sessions regarding anaemia. It is a matter of fact that there was no clear evidences or more details showing the number of health education sessions at UNRWA clinics in Gaza. From my special and family experiences at UNRWA clinics, the health education sessions are not sufficient to improve women perception and awareness about anaemia and focus only on individual level rather than in groups. The other explanation for that, the UNRWA antenatal clinics may be had shortage of qualified staff for that purpose. In addition, the sessions might be too short and seldom concentrating on specific issues on anaemia or may not be well-organized. The issue would be very interesting to be taken into account by UNRWA to reevaluate the strategy of health education sessions and the effectiveness of such sessions in terms of quality, quantity and scope. The study failed to demonstrate any difference in proportions of awareness of anaemia between women with and those without health education sessions except the statement regarding; adherence to have iron supplementation is necessary during pregnancy, this might be due to smaller sample size. As it is known that in a smaller sample size the differences would not be enough to be statistically significant.

The main strength of this study was that it was first of its nature and purposes, and it may provide useful evidences for decision makers to improve awareness and knowledge among pregnant women. In contrary, the significant limitation of the study was that, it consisted of a sample of only refugees women, so, the generalizability might be limited. The second weakness arises from its design as a cross-sectional study, as it is not possible to illustrate any conclusion about causation or effect. The other possible limitation was a smaller sample size which could be an absence of statistically significance.

\section{Conclusion}

The study should imply further examinations regarding the effectiveness and efficiency of health education at UNRWA antenatal health centers today. A clear strategy is needed to improve the health education sessions and make it more effective and productive. Finally, further studies are important to address this interesting area and it is recommended to include qualitative along with quantitative methods to deeply understand the phenomena. The next studies should also be extended to include a large sample of women and regions to assess other factors that may increase the increasing rate of anaemia among pregnant women in Palestine.

\section{Conflicts of Interest}

The authors declare that there is no conflicts of interest.

\section{Acknowledgements}

The authors would like to greatly thank everybody who involved and contributed in this work. The study had not received any funding.

\section{References}

[1] World health organization (WHO). The annual report of World health organization: reducing risks, promoting healthy life, WHO; 2002.

[2] N. Mikki, H. F. Abdul-Rahim, H. Stigum and G. HolmboeOsttesen. Anaemia prevalence and associated sociodemographic and dietary factors among Palestinian adolescents in the World Bank; 2011. Palestine.

[3] Knowledge and practices of maternity regarding anaemia and iron supplementation in Rural Malawi: a cross-sectional study. African Journal of Food Agriculture Nutrition and Development. 2009; 9 (1): 550-554. Available from: http://www.bioline.org.br/request?nd0900 (cited 17 May 2019)

[4] World health organization (WHO). Anaemia. WHO; 2010. Available from: http://www.who.int/topics/anaemia/en/(cited March 2019).

[5] World health organization (WHO). Iron deficiency anaemia: assessment, prevention, and control. A guide for program managers. Geneva. WHO; 2001 (WHO/NHD/01.3). 
[6] World health organization (WHO). United Nations Children Fund Report- Focusing on anaemia: towards and integrated approach for effective anaemia control. WHO and UNICEF; 2004.

[7] Minaz Mawani, Savera Aziz Ali, Gulshan Bano and Sumera Aziz Ali. Iron Deficiency Anemia among Women of Reproductive Age, an Important Public Health Problem: Situation Analysis. Reproductive System \& Sexual Disorders: Current Research. 2016; 5 (3). DOI: 10.4172/2161-038X. 1000187.

[8] A Taha, S Azhar, T Lone, G Murtaza, SA Khan, and others. Iron Deficiency Anaemia In Reproductive Age Women Attending Obstetrics And Gynecology Outpatient Of University Health Centre In Al-Ahsa, Saudi Arabia. African Journal of Traditional, Complementary and Alternative Medicines. 2014; 11 (2).

[9] Ministry of Palestinian health. (MOPH). Women health: health status in Palestine. MOH; 2016.

[10] Candio F., Hofmeyr GJ. Treatments of iron-deficiency anaemia among pregnant women. RHL commentary. The WHO Reproductive Health Library; Geneva: WHO 2007.

[11] Di Renzo, Spano, Giardina, Brillo, Clerici \& Roura. Iron Deficiency Anemia in Pregnancy. SAGE Journal, Women Health. 2015; 11 (6): 891-900. https://doi.org/10.2217/whe. 15.35 .

[12] Öztürk, Mustafa, et al. "Anemia prevalence at the time of pregnancy detection." Turkish journal of obstetrics and gynecology. 2017; 14 (3): 176.

[13] United Nations Relief and Work Agency (UNRWA). Study protocol on assessment of the prevalence of anaemia among pregnant women and children (6 months- 3years) of age. Palestine; 2006.

[14] World health organization (WHO). Worldwide prevalence of anaemia 1995-2005, Geneva: WHO; 2005.
[15] A Khader, H Madi, F Ricardo and G Sabatinelli. Anaemia among Palestinian pregnant women in Palestine. 2009; 12 (12): 2416-2420.

[16] Palestinian National Authority (PNA). Ministry of health report -nutrition department. National Nutrition Surveillance System 2009 Report. Palestine; 2009: 71.

[17] United Nations Relief and Work Agency (UNRWA). What We Do: Health. Available from: https://www.unrwa.org/what-wedo/health (cited 24 June 2019).

[18] United Nations Relief and Work Agency (UNRWA). Fact sheets. Jan. 2009.

[19] A. Daher, Rafael T., Mikolajczyk, Annette E., and others. Attitudes toward wife beating among Palestinian women of reproductive age from three cities in West Bank. Journal of Interpersonal Violence. 2010; 25 (3): 518-537.

[20] Food and agriculture organization of the United Nations. Palestine nutrition profile-food and nutrition division. FAO; 2005: 13-14.

[21] United Nations Relief and Work Agency (UNRWA). Annual report of the department of health. UNRWA; 2012.

[22] United Nations Relief and Work Agency (UNRWA). Epidemiological bulletin for Gaza strip. Palestine. 2010; 2 (4): 4.

[23] World health organization (WHO). Health condition in the Occupied Palestinian territory, including East Jerusalem, and in the occupied Syrian Jolan. WHO; 2009: 4. (A62/INF. DOC./2).

[24] Amani Waleed A. Iron deficiency anaemia among pregnant women in Nablus district: prevalence, knowledge, attitudes and practices. West Bank, Palestine; 2007: 31-37.

[25] Kristei Leong M. D. Featured health and wellness contributor. Available from: www.associatedcontent.com/article/2313687/the_side_effects _of_taking_iron_supplements.html (cited April 2019). 